\title{
Role of microRNA-130a in myocardial hypoxia/reoxygenation injury
}

\author{
HONGYAN LIU ${ }^{1}$, LEI HUAN ${ }^{1}$, JIE YIN ${ }^{1}$, MEILING QIN ${ }^{2}$, ZENGTANG ZHANG $^{1}$, \\ ZHIQIANG ZHANG ${ }^{1}$, JUNYE ZHANG ${ }^{3}$ and SHU WANG ${ }^{4}$ \\ ${ }^{1}$ Department of Cardiology, Laiwu City People's Hospital; ${ }^{2}$ Department of Endocrinology, \\ Traditional Chinese Medical Hospital of Laiwu, Laiwu, Shandong 271100; ${ }^{3}$ Cardiac Function Laboratory, Qilu Hospital of \\ Shandong University, Jinan, Shandong 250012; ${ }^{4}$ Sino-German Laboratory, Cardiovascular Institute and Fuwai Hospital, \\ Chinese Academy of Medical Science and Peking Union Medical College, Beijing 100037, P.R. China
}

Received September 7, 2015; Accepted October 5, 2016

DOI: $10.3892 /$ etm.2016.3984

\begin{abstract}
The aim of this study was to investigate the role of microRNA (miR)-130a in the pathogenesis of myocardial hypoxia/reoxygenation (H/R) injury. Primary rat cardiomyocytes were cultured and subjected to $H / R$ treatment. Reverse transcription-quantitative polymerase chain reaction was performed to detect the levels of miR-130a, western blot analysis was used to determine the expression of various proteins, and CCK- 8 assay was performed to determine cell viability. In addition, flow cytometry was used to assess apoptosis. The cell viability was significantly decreased and the apoptosis rate was significantly increased in H/R-treated primary cardiomyocytes, and the expression level of miR-130a was also elevated in these model cells. Transfection with miR-130a inhibitor significantly elevated the cell viability and reduced the apoptosis rate in $\mathrm{H} / \mathrm{R}$-treated cardiomyocytes. Bioinformatics analysis indicated that autophagy-related gene 14 (ATG14) is the target for miR-130a, which was confirmed by dual-luciferase reporter assay and western blot analysis. When the H/R model cells were co-transfected with miR-130a inhibitor and small interfering RNA against ATG14, the cell viability was significantly reduced and the apoptosis rate was significantly elevated, compared with that of cells transfected with miR-130a inhibitor alone. miR-130a inhibitor transfection significantly elevated the levels of ATG14 and phosphorylated (p-)Beclin 1, increased the LC3II/LC3I ratio, and decreased the expression levels of P62 and cleaved caspase-3, while the co-transfection of miR-130a inhibitor and siR-ATG14 attenuated these effects in H/R-induced primary cardiomyocytes. These results indicate that miR-130a is
\end{abstract}

Correspondence to: Dr Jie Yin, Department of Cardiology, Laiwu City People's Hospital, 1 Snow Lake Avenue, Laiwu, Shandong 271100, P.R. China

E-mail: yinjie006@163.com

Key words: microRNA, miR-130a, hypoxia/reoxygenation, autophagy-related gene 14, apoptosis, autophagy involved in H/R-induced injuries in primary cardiomyocytes, and that the inhibition of miR-130a increases the levels of ATG14 and p-Beclin 1, thereby increasing autophagy and inhibiting apoptosis in these cells.

\section{Introduction}

Myocardial ischemia/reperfusion (I/R) injury is a common pathological process observed in the clinic. I/R injury may be regulated by various factors, including oxidative stress, $\mathrm{Ca}^{2+}$ overload, myocardial energy metabolism disorder and cellular apoptosis (1). The occurrence of autophagy in myocardial cells during ischemia and hypoxia has been confirmed by numerous in vivo and in vitro studies, which might either promote the survival of myocardial cells or induce cell death $(2,3)$. It has been shown that the knockout of autophagy-related gene 5 (ATG5) in cardiac tissues may lead to spontaneous cardiac hypertrophy (2).

MicroRNA (miR) is a class of non-coding RNA, which is 18-22 nucleotides in length and participates in the regulation of cell proliferation, apoptosis and differentiation (4). In recent years, the effects of miR on the pathogenesis of heart diseases have attracted increasing attention. miR-130a has been shown to be expressed in the embryonic cardiac tissue, participating in the heart development process, and it has been suggested that upregulated miR-130a expression in embryonic cardiomyocytes might cause the ventricular muscle to be thinner and lead to abnormal heart structure (5). Moreover, miR-130a overexpression has been demonstrated to reduce the expression level of connexin 43, a major cardiac gap junction protein in mice, resulting in atrial and ventricular arrhythmias (6). In addition, it has been shown that the expression level of miR-130a is downregulated in various $\mathrm{I} / \mathrm{R}$ models $(7,8)$. However, the exact role of $\mathrm{miR}-130 \mathrm{a}$ in the pathogenesis of $\mathrm{I} / \mathrm{R}$ injury, particularly its association with autophagy, has not yet been fully elucidated.

In the present study, hypoxia/reoxygenation (H/R)-treated primary rat myocardial cells were used as an in vitro model for myocardial I/R injury, and the role of miR-130a in the pathogenesis of $H / R$ in myocardial cells, as well as the underlying mechanisms, were investigated. 


\section{Materials and methods}

Primary cell culture and identification. A total of 10 male Sprague-Dawley rats (age, 1-3 days) were provided by Shanghai Laboratory Animal Center (Shanghai, China). These rats were housed at $25^{\circ} \mathrm{C}$, under $50 \%$ humidity, feeding on normal diet. The animal experimental procedures were approved by the Ethics Committee of the Laiwu City People's Hospital (Laiwu, China). Following disinfection with $75 \%$ ethanol, the rats were sacrificed by cervical dislocation, and the ventricular muscle was removed. The tissue was cut into $1-\mathrm{mm}^{3}$ pieces, and digested with $0.25 \%$ trypsin in PBS buffer at $37^{\circ} \mathrm{C} 12-18$ times (5 min each time). The supernatant was collected, and Dulbecco's modified Eagle's medium (Gibco; Thermo Scientific, Inc., Waltham, MA, USA) containing 15\% FBS (Gibco; Thermo Scientific, Inc.) was added to stop the digestion. The cells were harvested using centrifugation at $800 \mathrm{x} g$ at room temperature for $10 \mathrm{~min}$, and re-suspended in fresh culture medium. The cardiomyocytes were purified using the differential adhesion separation method (9), and $100 \mu \mathrm{mol} / 1$ bromodeoxyuridine was used to inhibit the proliferation of other cells. After 72-h culture, these cardiomyocytes were used in the following experiments.

Cell transfection. The miR-130a mimics, miR-130a inhibitor, negative control (NC) and riboFECT ${ }^{\mathrm{TM}} \mathrm{CP}$ transfection reagent were purchased from Guangzhou RiboBio Co., Ltd. (Guangzhou, China). The small interfering RNA (siRNA) and NC sequence for ATG14 were purchased from Shanghai GenePharma Co., Ltd. (Shanghai, China). The primary cardiomyocytes were first incubated with serum-free medium for $12 \mathrm{~h}$, and then transfection was performed using Lipofectamine 2000 (Invitrogen; Thermo Scientific, Inc.), according to the manufacturer's instructions. Different concentrations of siRNA $(25,50$ and $100 \mathrm{nM})$ were used for ATG14 inhibition and $100 \mathrm{nM}$ siR-ATG14 was chosen for the following experiments. Control and H/R myocytes were transfected with $\mathrm{NC}$ sequences.

$H / R$ induction. At $24 \mathrm{~h}$ after transfection, the cardiomyocytes were subjected to $\mathrm{H} / \mathrm{R}$ induction, following previously published protocols $(10,11)$. These cells were cultured with serum-free medium in a $95 \% \mathrm{~N}_{2}$ plus $5 \% \mathrm{CO}_{2}$ atmosphere at $37^{\circ} \mathrm{C}$ for $6 \mathrm{~h}$, and then incubated with normal medium in a $95 \%$ air plus $5 \% \mathrm{CO}_{2}$ atmosphere at $37^{\circ} \mathrm{C}$ for $24 \mathrm{~h}$. The procedures were repeated three times.

Reverse transcription-quantitative polymerase chain reaction $(R T-q P C R)$. Total RNA was extracted from the cardiomyocytes with the RNeasy Mini kit (Qiagen, Inc., Valencia, CA, USA). Reverse transcription (Toyobo, Tokyo, Japan) was performed to synthesize the cDNA template, with $500 \mathrm{ng}$ RNA, $4 \mathrm{ml} 5 \mathrm{X}$ Mix, and $\mathrm{ddH}_{2} \mathrm{O}$ (to $20 \mathrm{ml}$ ), according to the following conditions: $37^{\circ} \mathrm{C}$ for $15 \mathrm{~min}, 50^{\circ} \mathrm{C}$ for $5 \mathrm{~min}$, and $98^{\circ} \mathrm{C}$ for $5 \mathrm{~min}$. qPCR was performed using a QuantiNova SYBR Green RT-PCR kit from Qiagen. The primers for miR-130a and U6 were purchased from Guangzhou Fulengen Co., Ltd. (Guangzhou, China). The primer sequences were as follows: miR-130a, CAGUGCAAUGUUAAAAG; U6 forward, 5'-CTCGCTTCG GCAGCACA-3' and reverse, 5'-ACGCTTCACGAATTTGCG
T-3'; ATG14 forward, 5'-ACCAGCATTAGCATCACG-3' and reverse, 5'-AGGTCCTTGGGTTGTTTT-3'; GAPDH forward, 5'-CCTCAAGATTGTCAGCAAT-3' and reverse, 5'-CCA TCCACAGTCTTCTGAGT-3'. The PCR conditions consisted of denaturation at $95^{\circ} \mathrm{C}$ for $1 \mathrm{~min} ; 95^{\circ} \mathrm{C}$ for $15 \mathrm{sec}, 60^{\circ} \mathrm{C}$ for $30 \mathrm{sec}$ and $72^{\circ} \mathrm{C}$ for $15 \mathrm{sec}$, for 40 cycles; and a final extension at $72^{\circ} \mathrm{C}$ for $1 \mathrm{~min}$. The relative expression levels of target genes were calculated with the $2^{-\Delta \Delta C q}$ method (12). Negative controls were set up using $\mathrm{ddH}_{2} \mathrm{O}$ instead of template. Experiment was performed in triplicates.

Western blot analysis.Cardiomyocytes were collected and lysed with the RIPA lysis buffer (Beyotime Institute of Biotechnology, Haimen, China) on ice for $15 \mathrm{~min}$. Following centrifugation at $12,000 \mathrm{x} \mathrm{g}$ at $4^{\circ} \mathrm{C}$ for $10 \mathrm{~min}$, the protein concentration was determined using the BCA method. A 30- $\mu \mathrm{g}$ sample of protein was separated with $10 \%$ SDS-PAGE, and transferred onto a polyvinylidene difluoride membrane. The membrane was then blocked with $5 \%$ fat-free milk at room temperature for $1 \mathrm{~h}$, and incubated with rabbit anti-ATG14 primary antibody (BS8969; 1:1,000; Bioworld Technology, Inc., Minneapolis, MN, USA), rabbit anti-phosphorylated-Beclin 1 primary antibody (84966; 1:1,000; Cell Signaling Technology, Inc., Beverly, CA, USA), rabbit anti-LC3 primary antibody (AL221; 1:1,000; Beyotime Institute of Biotechnology), rabbit anti-P62 primary antibody (5114), rabbit anti-cleaved caspase-3 primary antibody (9661; both 1:1,000; Cell Signaling Technology, Inc.), and mouse anti-GAPDH primary antibody (BS60630; 1:2,000; Bioworld Technology, Inc.), respectively, at $4^{\circ} \mathrm{C}$ overnight. Subsequently, the membrane was incubated with goat anti-rabbit secondary antibody (BS13271) and goat anti-mouse secondary antibody (BS12471; both 1:5,000; Bioworld Technology, Inc.), respectively, at room temperature for $1 \mathrm{~h}$. The blot was developed with the ECL method (Beyotime Institute of Biotechnology, Haimen, China). Protein bands were detected using Quantity One software (Bio Rad Laboratories, Inc., Hercules, CA, USA).

Cell viability assay. The viability of cardiomyocytes was assessed with the CCK-8 assay kit (Beyotime Institute of Biotechnology). The primary cells were planted onto a 96-well plate. After transfection, $10 \mu \mathrm{l}$ CCK- 8 was added and the cells were incubated in a $37^{\circ} \mathrm{C}, 5 \% \mathrm{CO}_{2}$ atmosphere for $1 \mathrm{~h}$. The absorbance at $450 \mathrm{~nm}$ was then read with a microplate reader (Thermo Fisher Scientific, Inc.). The cell viability was calculated according to the manufacturer's instructions.

Flow cytometry. The apoptotic process was evaluated with flow cytometry. Following digestion with $1 \mathrm{ml}$ Trypsin (Gibco; Thermo Fisher Scientific, Inc., Grand Island, NY, USA) at $37^{\circ} \mathrm{C}$ for $3 \mathrm{~min}$ and washing, the cardiomyocytes were incubated with $100 \mu \mathrm{l}$ binding buffer, $5 \mu \mathrm{l}$ Annexin V/fluorescein isothiocyanate and $5 \mu \mathrm{l}$ propidium iodide in the dark at room temperature for $15 \mathrm{~min}$. After $1 \mathrm{~h}$, the fluorescence was detected using a flow cytometer (BD FACSCalibur; BD Biosciences, Franklin Lakes, NJ, USA) and the apoptosis rate was calculated using BD FACSuite software (BD Biosciences).

Dual-luciferase reporter assay. The potential target sequences for miR-130a in the 3'-untranslated region (3'-UTR) of ATG14 and the mutated sequences of this $3^{\prime}$-UTR were as follows: 
A

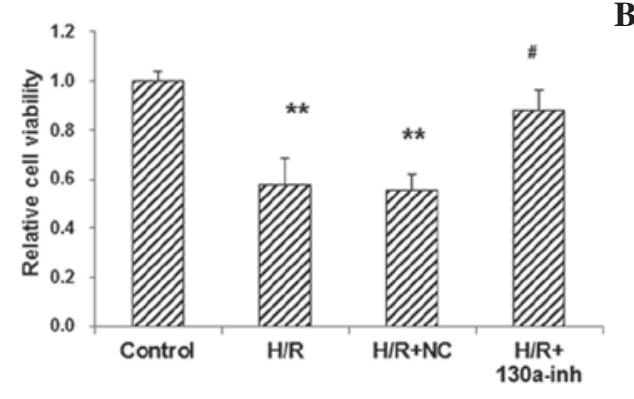

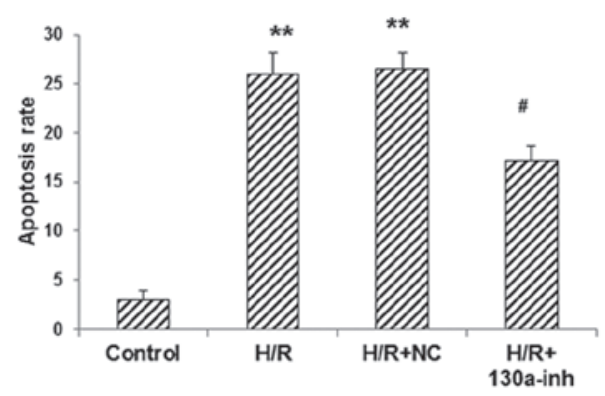

C

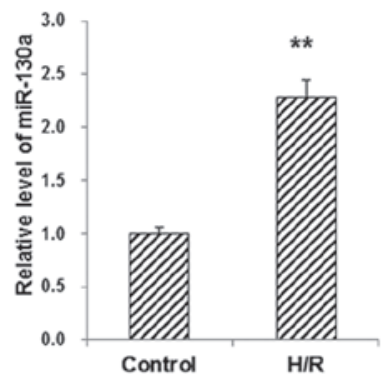

D

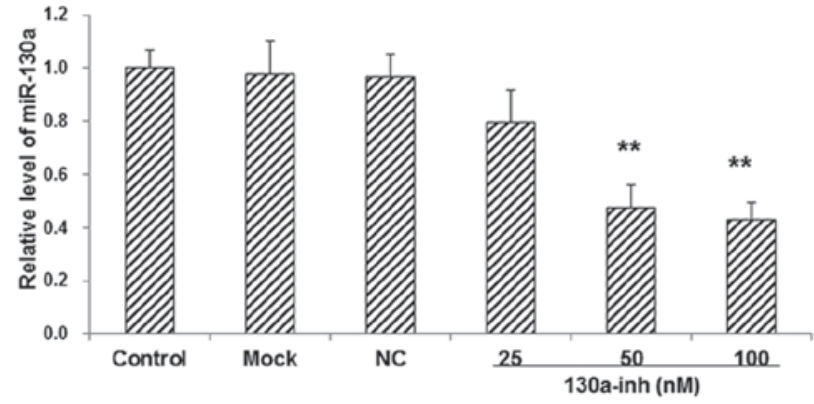

$\mathbf{E}$
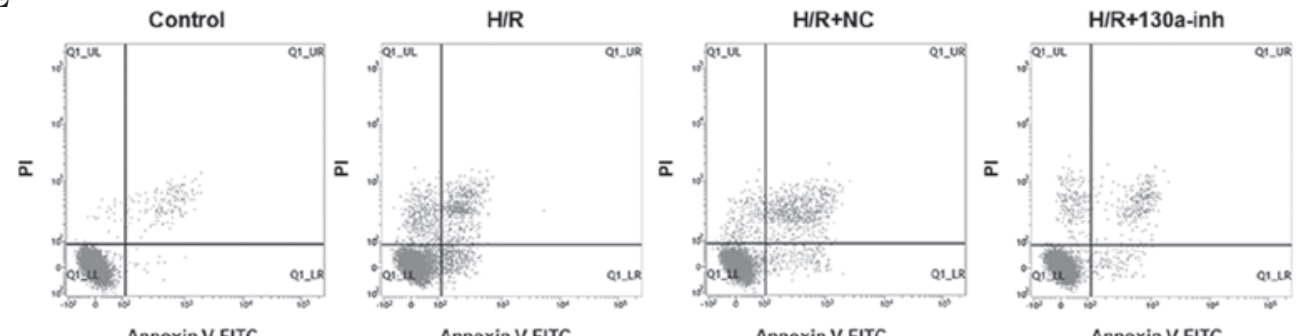

Figure 1. Involvement of miR-130a in H/R-induced injuries in primary cardiomyocytes. (A) Cell viability and (B) apoptosis of H/R-induced cardiomyocytes transfected with $100 \mathrm{nM}$ miR-130a inhibitor were assessed. (C) The expression level of miR-130a in H/R-induced cardiomyocytes was detected using RT-qPCR. (D) Primary cardiomyocytes were transfected with miR-130a inhibitor at 25, 50, and $100 \mathrm{nM}$, respectively, and RT-qPCR was performed to detect the expression levels of miR-130a in these cells. (E) Representative results for the flow cytometry to detect apoptosis. ${ }^{* *} \mathrm{P}<0.01$ vs. the normal control group; ${ }^{\text {"}} \mathrm{P}<0.05$ vs. H/R-induced model cells. miR, microRNA; H/R, hypoxia/reoxygenation; RT-qPCR, reverse transcription-quantitative polymerase chain reaction; $\mathrm{NC}$, negative control; inh, inhibitor.

Wild-type ATG14 3'-UTR, 5'-AUUUUGCACUG-3'; and mutant ATG14 3'-UTR: 5'-AGUCUACGCAG-3'. These sequences were amplified, and cloned into psiCHECK ${ }^{\mathrm{TM}}$ reporter plasmids (Promega Corporation, Madison, WI, USA) to obtain wt-ATG14-3'-UTR and mut-ATG14-3'-UTR plasmids. The reconstructed plasmids were co-transfected with miR-130a or NC mimics into 293T cells from the Cell Bank of the Chinese Academy of Sciences (Shanghai, China). Results were obtained and analyzed according to the manufacturer's instructions. All experiments were repeated three times independently.

Bioinformatics analysis. Target genes of miR 130a were predicted using TargetScan software (www.targetscan.org).

Statistical analysis. Data were expressed as mean \pm standard deviation. SPSS version 19.0 software was used for statistical analysis (IBM SPSS, Armonk, NY, USA). The $\mathrm{t}$-test was used for pair-wise comparison, while analysis of variance was performed for multiple comparison. $\mathrm{P}<0.05$ was considered to indicate a statistically significant difference.

\section{Results}

$H / R$ decreases cell viability and increases apoptosis in primary cardiomyocytes. To investigate the effects of $\mathrm{H} / \mathrm{R}$ treatment on primary cardiomyocytes, cell viability and apoptosis were detected with CCK-8 assay and flow cytometry, respectively. The results from the CCK- 8 assay showed that, compared with the control group, cell viability was significantly decreased in primary cardiomyocytes following $\mathrm{H} / \mathrm{R}$ treatment $(\mathrm{P}<0.01$; Fig. 1A). Moreover, the results from flow cytometry showed that, compared with the control group, the apoptosis rate was significantly increased in H/R-induced primary cardiomyocytes $(\mathrm{P}<0.01$; Fig. 1B). These results indicate that the $H / R$ treatment significantly decreased the cell viability and increased the apoptosis rate of primary cardiomyocytes.

miR-130a participates in $H / R$-induced injuries in primary cardiomyocytes. The expression level of miR-130a in H/R-induced cardiomyocytes was detected with RT-qPCR. The results showed that, compared with the control group, the expression level of miR-130a was significantly elevated in primary cardiomyocytes following $\mathrm{H} / \mathrm{R}$ treatment $(\mathrm{P}<0.01$; 
A 3' UACGgGaAaAUUGUAACGUGaC miR-130a | ||||||||

5'... UGGGAUUUCGUUAUUUUGCACUG... W. Wt-ATG14 3'-UTR

5'...UGGGAUUUCGUUAUUAACGUGCA... mut-ATG14 3'-UTR

B

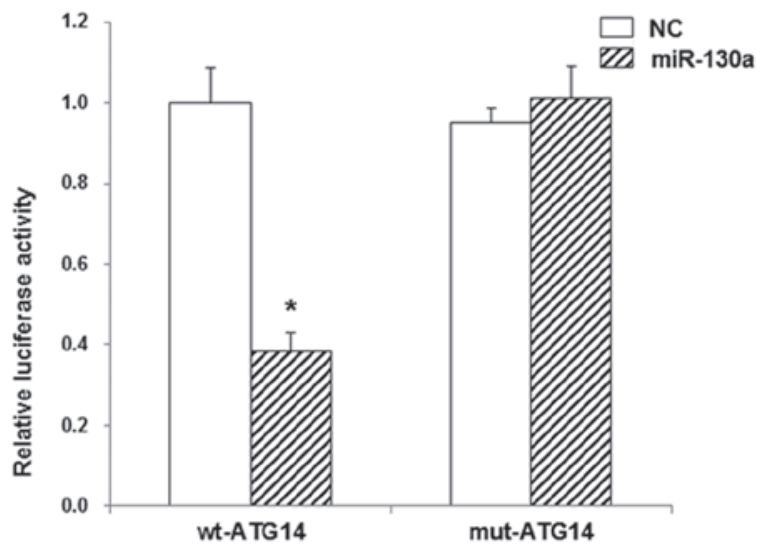

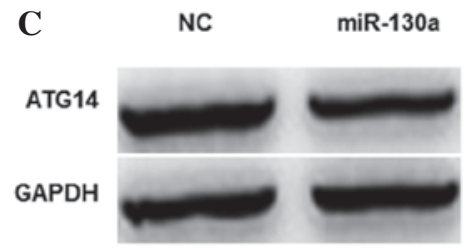

Figure 2. Interaction between miR-130a and ATG14. (A) Prediction of miR-130a target gene with TargetScan software. (B) Dual-luciferase reporter assay results showing the interaction between miR-130a and ATG14. (C) The expression levels of ATG14 as detected by western blot analysis. "P<0.01 vs. the NC group. miR, microRNA; ATG14, autophagy-related gene 14; UTR, untranslated region; wt, wild type; mut, mutant; NC, negative control.

Fig. 1C), indicating that miR-130a might be associated with the pathogenic process of H/R-induce injury.

To further investigate the role of miR-130a in the pathogenesis of H/R-induced injuries in primary cardiomyocytes, these cells were transfected with miR-130a inhibitor, and RT-qPCR was performed to confirm the manipulation. The results showed that, compared with the control group, transfection with miR-130a inhibitor $(25,50$ and $100 \mathrm{nM})$ significantly decreased the expression levels of miR-130a in primary cardiomyocytes in a dose-dependent manner $(\mathrm{P}<0.01$; Fig. 1D). When the H/R-induced cardiomyocytes were transfected with $100 \mathrm{nM}$ miR-130a inhibitor, the cell viability was significantly elevated $(\mathrm{P}<0.05$; Fig. $1 \mathrm{~A})$, while the apoptosis rate was significantly reduced $(\mathrm{P}<0.05$; Fig. $1 \mathrm{~B}$ and $\mathrm{E})$ in these H/R model cells. These results suggest that miR-130a is involved in $\mathrm{H} / \mathrm{R}$-induced injuries in primary cardiomyocytes.

miR-130a targets ATG14 in H/R-induced primary cardiomyocytes. Target genes of miR-130a were predicted with TargetScan software. The results indicated that miR-130 might bind to the 3'-UTR of ATG14 at 1299-1306 bp (Fig. 2A). The dual-luciferase reporter assay was then performed to investigate the interaction between miR-130a and ATG14. The results showed that when the miR-130a mimics and the reporter plasmid containing the wild-type 3'-UTR of ATG14 were co-transfected into the $293 \mathrm{~T}$ cells the luminescence intensity was significantly reduced by $38 \%(\mathrm{P}<0.05)$. By contrast, in the cells co-transfected with miR-130a mimics and the luciferase reporter plasmid containing the mutant 3'-UTR of ATG14, no significant differences were observed in the luminescence intensity ( $\mathrm{P}>0.05$; Fig. $2 \mathrm{~B}$ ). To further confirm this interaction, western blot analysis was performed. The results showed that, compared with the control group, transfection with miR-130a mimics clearly reduced the protein expression level of ATG14 (Fig. 2C). These results suggest that miR-130a targets ATG14 in the 3'-UTR and inhibits the gene expression.
To investigate the involvement of ATG14 in the pathogenesis of H/R-induced injuries, the expression of ATG14 was manipulated in primary cardiomyocytes. RT-qPCR showed that, compared with the control group, transfection with siR-ATG14 $(25,50$ and $100 \mathrm{nM}$, respectively) significantly decreased the mRNA expression levels of ATG14 in primary cardiomyocytes in a dose-dependent manner $(\mathrm{P}<0.01$; Fig. 3A). The H/R-induced primary cardiomyocytes were then co-transfected with miR-130a inhibitor and siR-ATG14. The results from the CCK-8 assay and flow cytometry showed that, compared with the miR-130a-treated H/R models, siR-ATG14 significantly reduced the cell viability $(\mathrm{P}<0.05$; Fig. $3 \mathrm{~B})$ and elevated the apoptosis rate $(\mathrm{P}<0.05$; Fig. $3 \mathrm{C}$ and $\mathrm{D})$, in these cells. These results suggest that the knockdown of ATG14 blocked the protective effects of miR-130a inhibitor on $\mathrm{H} / \mathrm{R}$-induced primary cardiomyocytes, which confirms the involvement of ATG14 in the miR-130a-mediated H/R injuries in these cells.

miR-130a inhibition enhances autophagy in H/R-induced primary cardiomyocytes. ATG14 plays an important role in the initiation of autophagy (13). Therefore, the autophagic process in the H/R-induced primary cardiomyocytes was investigated in the present study. The results of western blot analysis showed that, compared with the H/R-induced model cells, the transfection with miR-130a inhibitor clearly elevated the expression levels of ATG14 and phosphorylated Beclin 1 (p-Beclin 1), increased the ratio of LC3II/LC3I, and decreased the expression levels of P62 (Fig. 4), in the H/R-induced primary cardiomyocytes, indicating increased autophagy. In addition, miR-130a inhibitor transfection also decreased the expression levels of cleaved caspase-3 in the H/R-induced primary cardiomyocytes (Fig. 4), further confirming that miR-103a inhibitor restrained the apoptotic process induced by $H / R$ in these cells. However, co-transfection with miR-130a inhibitor and siR-ATG14 attenuated the changes in 
A

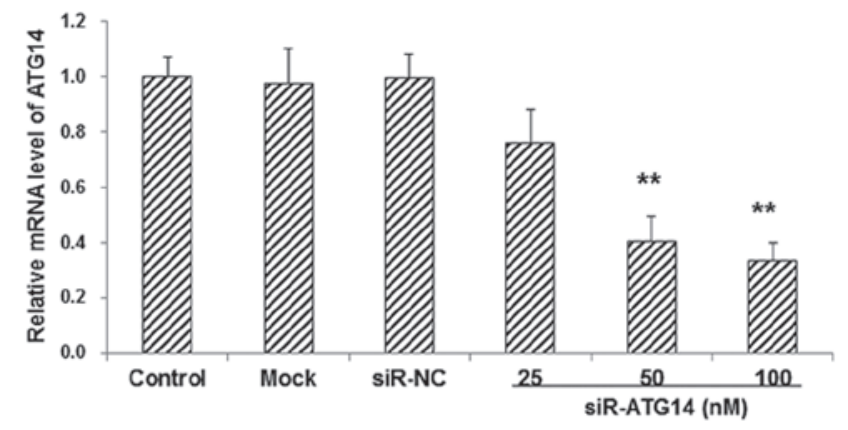

B

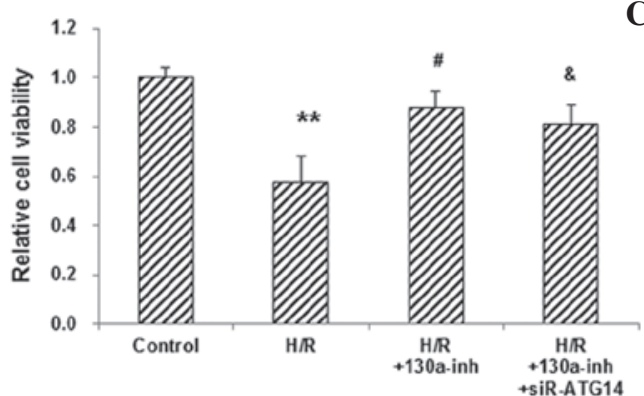

C

D
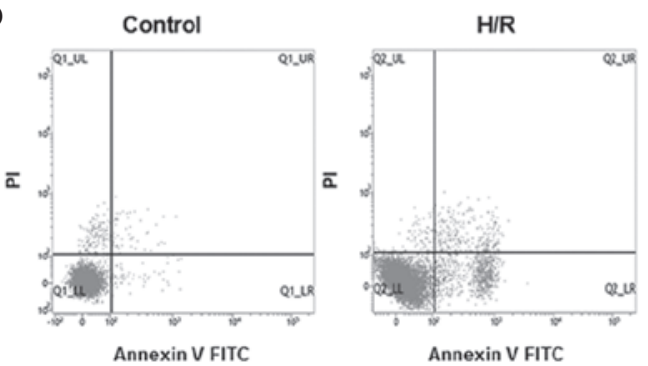

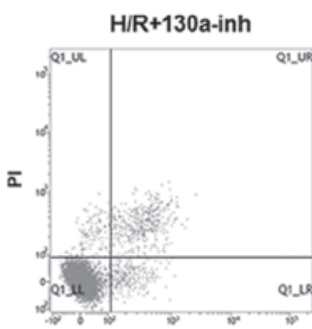

Annexin V FITC

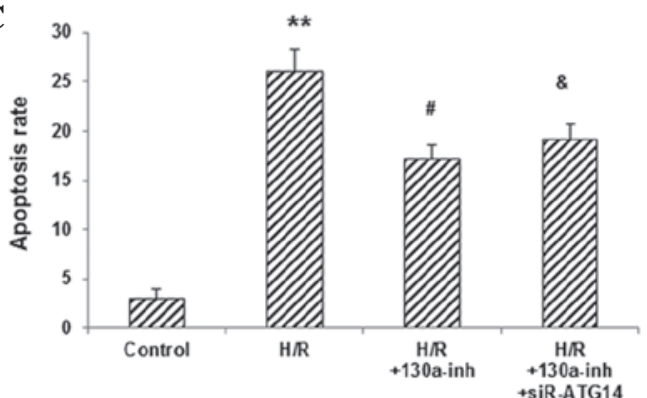

Figure 3. Effects of ATG14 interference on cell viability and apoptosis in H/R-induced primary cardiomyocytes. (A) Primary cardiomyocytes were transfected with siR-ATG14 at 25, 50, and $100 \mathrm{nM}$, respectively, and the mRNA expression levels of ATG14 were detected with RT-qPCR. The H/R-induced primary cardiomyocytes were co-transfected with miR-130a inhibitor and siR-ATG14, and then (B) the cell viability and (C) apoptosis were assessed using CCK-8 assay and flow cytometry, respectively. (D) Representative results for the flow cytometric detection of apoptosis. ${ }^{* *} \mathrm{P}<0.01$ vs. the $\mathrm{NC}$ group; ${ }^{\#} \mathrm{P}<0.05$ vs. the H/R-induced model cells; ${ }^{\&} \mathrm{P}<0.05$ vs. the H/R models treated with miR-130a inhibitor. ATG14, autophagy-related gene 14; H/R, hypoxia/reoxygenation; RT-qPCR, reverse transcription-quantitative polymerase chain reaction; siR, small interfering RNA; NC, negative control; inh, inhibitor.

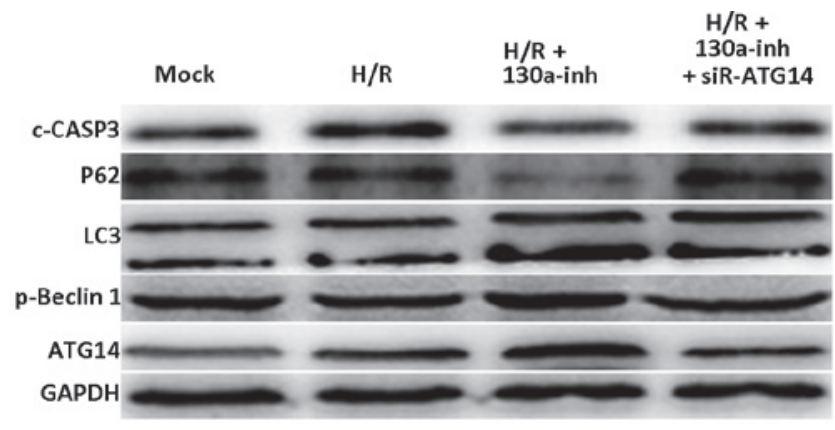

Figure 4. Effects of miR-130a inhibition on autophagy and apoptosis in $\mathrm{H} / \mathrm{R}$-induced primary cardiomyocytes. Western blot analysis was performed to detect the expression levels of ATG14, p-Beclin 1, LC3, P62 and c-CASP3 in the H/R-induced primary cardiomyocytes transfected with miR-130a inhibitor and/or siR-ATG14. miR, microRNA; H/R, hypoxia/reoxygenation ATG14, autophagy-related gene 14; p-Beclin 1, phosphorylated Beclin 1; c-CASP3, cleaved caspase-3; inh, inhibitor.

the expression levels of p-Beclin 1, LC3II, P62 and cleaved caspase-3 in H/R-induced primary cardiomyocytes (Fig. 4). These results suggest that the miR-130a inhibitor regulated the expression levels of ATG14 and increased autophagy in primary cardiomyocytes, thereby protecting these cells from the apoptosis induced by $\mathrm{H} / \mathrm{R}$ treatment.

\section{Discussion}

Abnormal expression of various miRs has been reported in the pathogenesis of cardiovascular diseases. Recent studies have found that $\mathrm{miR}$ is involved in apoptosis and other injuries in myocardial cells caused by I/R (H/R) (14-16). Liu et al (17) have shown that miR-15b increases I/R-induced myocardial apoptosis through the mitochondrial pathway. Moreover, Wang et al (18) found that miR-494 is able to regulate antiand pro-apoptotic proteins to protect myocardial cells from the I/R-induced apoptosis. Furthermore, inhibition of miR-92a has been found to reduce the I/R-induced apoptosis and necrosis of myocardial cells (19). In addition, Jakob et al (20) have shown that the expression of miR-130a is downregulated in endothelial outgrowth cells and CD $34^{+}$cells in patients with chronic heart failure caused by ischemic heart disease, and miR-130a overexpression increases cell activities, promotes angiogenesis, and improves cardiac function. It has also been shown that miR-130a is upregulated in the peripheral blood of patients with coronary heart disease, indicating an increased 
risk of cardiovascular events (21). Jegga et al (22) also demonstrated an association between miR-130a and cellular autophagy regulation.

In the present study, the results showed that the expression levels of miR-130a in primary rat cardiomyocytes were elevated by H/R treatment. TargetScan analysis indicated that ATG14 is one of the targets of miR-130a. Therefore, the effects of miR-130a inhibitor and siR-ATG14 on the $\mathrm{H} / \mathrm{R}$-induced primary rat cardiomyocytes were investigated. It was found that miR-130a inhibitor transfection improved the survival and decreased the apoptosis of cardiomyocytes, which was blocked by co-transfection with siR-ATG14. The dual-luciferase reporter assay was then performed to further investigate whether ATG14 was the target gene for miR-130a. The results showed that co-transfection with miR-130a mimics and reporter plasmid containing the wild-type 3'-UTR of ATG14 resulted in a significant reduction in the luminescence intensity. However, in the cells co-transfected with miR-130a mimics and luciferase reporter plasmid containing the mutant 3'-UTR of ATG14, no significant differences in the luminescence intensity were observed. Moreover, western blot analysis showed that the expression level of ATG14 was decreased following transfection with miR-130a mimics. These results suggest that ATG14 is the target gene for miR-130a, and that inhibition of miR-130a regulated the expression level of ATG14 and increased autophagy to protect the cardiomyocytes against H/R-induced apoptosis and improve cell survival.

ATG14, which is also known as Beclin 1-associated autophagy-related key regulator(Barkor), is located in the endoplasmic reticulum, isolation membrane and autophagosomes. ATG14 is able to increase the activity of Vps34 and plays an important role in the initiation of the autophagic process, and mutation of ATG14 results in attenuated autophagy $(23,24)$. Fogel et al (25) have shown that ATG14 regulates the phosphorylation of Beclin 1, which is a core component of the phosphatidylinositol-3 complex and also plays an important role in the occurrence of autophagy. Consistent with this, the results of the present study showed that the increased expression of ATG14 was accompanied by the elevated expression of p-Beclin 1. LC3II and P62 are autophagic markers, and the LC3II/LC3I ratio reflects autophagic activity (26). The results of the present study showed that following transfection with miR-130a inhibitor, the expression of LC3II was elevated while the level of cleaved caspase- 3 was reduced in the primary cardiomyocytes. However, transfection with ATG14 decreased the expression levels of p-Beclin 1 and LC3II, indicating that autophagic activity was inhibited, but increased the expression of cleaved caspase-3, indicating increased apoptosis in these cells. The aforementioned results suggest that ATG14 affects the phosphorylation of Beclin 1 and regulates apoptotic and autophagic cell death in primary cardiomyocytes.

In conclusion, the results of the present study showed that the expression levels of miR-130a were elevated in H/R-treated primary rat cardiomyocytes, and this was associated with the pathogenesis of H/R-induced injury. The inhibition of miR-130a increased the expression level of ATG14 and the phosphorylation level of Beclin 1, therefore increasing autophagy and inhibiting apoptosis, in the H/R-induced primary cardiomyocytes. These findings might help to increase understanding of the roles of miR-130a and ATG14 in the pathogenesis of $\mathrm{H} / \mathrm{R}$-induced injuries.

\section{Acknowledgements}

The authors would like to thank Professor Mei Zhang in Qilu Hospital, Shandong University, China for help during this study.

\section{References}

1. Frangogiannis NG: Regulation of the inflammatory response in cardiac repair. Circ Res 110: 159-173, 2012.

2. Przyklenk K, Undyala VV, Wider J, Sala-Mercado JA, Gottlieb RA and Mentzer RM Jr: Acute induction of autophagy as a novel strategy for cardioprotection: Getting to the heart of the matter. Autophagy 7: 432-433, 2011.

3. Yue HW, Liu J, Liu PP, Li WJ, Chang F, Miao JY and Zhao J: Sphingosylphosphorylcholine protects cardiomyocytes against ischemic apoptosis via lipid raft/PTEN/Akt1/mTOR mediated autophagy. Biochim Biophys Acta 1851: 1186-1193, 2015.

4. He XM, Zheng YQ, Liu SZ, Liu Y, He YZ and Zhou XY: Altered Plasma MicroRNAs as Novel Biomarkers for Arteriosclerosis Obliterans. J Atheroscler Thromb 23: 196-206, 2016.

5. Kim GH, Samant SA, Earley JU and Svensson EC: Translational control of FOG-2 expression in cardiomyocytes by microRNA-130a. PLoS One 4: e6161, 2009.

6. Osbourne A, Calway T, Broman M, McSharry S, Earley J and Kim GH: Downregulation of connexin43 by microRNA-130a in cardiomyocytes results in cardiac arrhythmias. J Mol Cell Cardiol 74: 53-63, 2014.

7. Ye Y, Perez-Polo JR, Qian J and Birnbaum Y: The role of microRNA in modulating myocardial ischemia-reperfusion injury. Physiol Genomics 43: 534-542, 2011.

8. Dong S, Cheng Y, Yang J, Li J, Liu X, Wang X, Wang D, Krall TJ, Delphin ES and Zhang C: MicroRNA expression signature and the role of microRNA-21 in the early phase of acute myocardial infarction. J Biol Chem 284: 29514-29525, 2009.

9. Xu W, Jiang $\mathrm{H}, \mathrm{Hu} \mathrm{X}$ and $\mathrm{Fu} \mathrm{W}$ : Effects of high-mobility group box 1 on the expression of Beclin-1 and LC3 proteins following hypoxia and reoxygenation injury in rat cardiomyocytes. Int J Clin Exp Med 7: 5353-5357, 2014.

10. Wang C, Li YZ, Wang XR, Lu ZR, Shi DZ and Liu XH: Panax quinquefolium saponins reduce myocardial hypoxia-reoxygenation injury by inhibiting excessive endoplasmic reticulum stress. Shock 37: 228-233, 2012.

11. Li Y, Ge X and Liu X: The cardioprotective effect of postconditioning is mediated by ARC through inhibiting mitochondrial apoptotic pathway. Apoptosis 14: 164-172, 2009.

12. Livak KJ and Schmittgen TD: Analysis of relative gene expression data using real-time quantitative PCR and the 2(-Delta Delta C(T)) Method. Methods 25: 402-408, 2001.

13. Liu R, Zhi X and Zhong Q: ATG14 controls SNARE-mediated autophagosome fusion with a lysosome. Autophagy 11: 847-849, 2015.

14. Qian L, Van Laake LW, Huang Y, Liu S, Wendland MF and Srivastava D: miR-24 inhibits apoptosis and represses Bim in mouse cardiomyocytes. J Exp Med 208: 549-560, 2011.

15. Song CL, Liu B, Diao HY, Shi YF, Li YX, Zhang JC, Lu Y, Wang G, Liu J, Yu YP, et al: The protective effect of microRNA-320 on left ventricular remodeling after myocardial ischemia-reperfusion injury in the rat model. Int J Mol Sci 15: 17442-17456, 2014.

16. Yu H, Seo JB, Jung SR, Koh DS and Hille B: Noradrenaline upregulates T-type calcium channels in rat pinealocytes. J Physiol 593: 887-904, 2015.

17. Liu L, Zhang G, Liang Z, Liu X, Li T, Fan J, Bai J and Wang Y: MicroRNA-15b enhances hypoxia/reoxygenation-induced apoptosis of cardiomyocytes via a mitochondrial apoptotic pathway. Apoptosis 19: 19-29, 2014.

18. Wang X, Zhang X, Ren XP, Chen J, Liu H, Yang J, Medvedovic M, Hu Z and Fan GC: MicroRNA-494 targeting both proapoptotic and antiapoptotic proteins protects against ischemia/reperfusion-induced cardiac injury. Circulation 122: 1308-1318, 2010. 
19. Hinkel R, Penzkofer D, Zühlke S, Fischer A, Husada W, Xu QF Baloch E, van Rooij E, Zeiher AM, Kupatt C and Dimmeler S: Inhibition of microRNA-92a protects against ischemia/ reperfusion injury in a large-animal model. Circulation 128: 1066-1075, 2013

20. Jakob P, Doerries C, Briand S, Mocharla P, Kränkel N, Besler C, Mueller M, Manes C, Templin C, Baltes C, et al: Loss of angiomiR-126 and 130a in angiogenic early outgrowth cells from patients with chronic heart failure: Role for impaired in vivo neovascularization and cardiac repair capacity. Circulation 126: 2962-2975, 2012.

21. Jansen F, Yang X, Proebsting S, Hoelscher M, Przybilla D, Baumann K, Schmitz T, Dolf A, Endl E, Franklin BS, et al: MicroRNA expression in circulating microvesicles predicts cardiovascular events in patients with coronary artery disease. J Am Heart Assoc 3: e001249, 2014
22. Jegga AG, Schneider L, Ouyang X and Zhang J: Systems biology of the autophagy-lysosomal pathway. Autophagy 7: 477-489, 2011.

23. Zhao Z, Tao L, Shen C, Liu B, Yang Z and Tao H: Silencing of Barkor/ATG14 sensitizes osteosarcoma cells to cisplatin-induced apoptosis. Int J Mol Med 33: 271-276, 2014.

24. Matsunaga K, Morita E, Saitoh T, Akira S, Ktistakis NT, Izumi T, Noda T and Yoshimori T: Autophagy requires endoplasmic reticulum targeting of the PI3-kinase complex via Atg14L. J Cell Biol 190: 511-521, 2010.

25. Fogel AI, Dlouhy BJ, Wang C, Ryu SW, Neutzner A, Hasson SA, Sideris DP, Abeliovich $\mathrm{H}$ and Youle RJ: Role of membrane association and Atg14-dependent phosphorylation in beclin-1-mediated autophagy. Mol Cell Biol 33: 3675-3688, 2013.

26. Morgan MJ and Thorburn A: Measuring Autophagy in the Context of Cancer. Adv Exp Med Biol 899: 121-143, 2016. 